\title{
The hidden competing phase revealed by first-principles calculations of phonon instability in the nearly optimally doped cuprate $\mathrm{La}_{1.875} \mathrm{Sr}_{0.125} \mathrm{CuO}_{4}$
}

\author{
Chi-Cheng Lee, ${ }^{1,2}$ Ji-Yao Chiu, ${ }^{1}$ Yukiko Yamada-Takamura, ${ }^{3}$ and Taisuke Ozaki ${ }^{4}$ \\ ${ }^{1}$ Department of Physics, Tamkang University, Tamsui, New Taipei 251301, Taiwan \\ ${ }^{2}$ Research Center for X-ray Science, College of Science, \\ Tamkang University, Tamsui, New Taipei 251301, Taiwan \\ ${ }^{3}$ School of Materials Science, Japan Advanced Institute of Science and \\ Technology (JAIST), 1-1 Asahidai, Nomi, Ishikawa 923-1292, Japan \\ ${ }^{4}$ Institute for Solid State Physics, The University of Tokyo, \\ 5-1-5 Kashiwanoha, Kashiwa, Chiba 277-8581, Japan
}

(Dated: July 12, 2021)

\begin{abstract}
The representative cuprate, $\mathrm{La}_{2-x} \mathrm{M}_{x} \mathrm{CuO}_{4}$, with $\mathrm{M}=\mathrm{Sr}$ and $x=1 / 8$ is studied via first-principles calculations in the high-temperature tetragonal (HTT), low-temperature orthorhombic (LTO), and low-temperature less-orthorhombic (LTLO) structures. By suppressing the magnetism and superconductivity, the LTLO phase, which has rarely been observed in $\mathrm{La}_{2-x} \mathrm{Sr}_{x} \mathrm{CuO}_{4}$, is found to be the ground state, where the structural phase transitions, HTT $\rightarrow$ LTO $\rightarrow$ LTLO, can be understood via phonon instability. While the La-O composition is identified to be responsible for the phonon softening, the superconducting $\mathrm{CuO}_{2}$ layer is dynamically stable. The LTLO phase, which can exhibit $\mathrm{a} \sim 20 \mathrm{meV}$ splitting in the density of states, is proposed to have an intimate relationship with the observed pseudogap and the charge density wave giving the stripe. We argue that at low temperatures, the superconducting LTO $\mathrm{La}_{1.875} \mathrm{Sr}_{0.125} \mathrm{CuO}_{4}$ competes with the phonon-preferred LTLO phase by spontaneously forming the Cooper pairs, resulting in suppressing the stripe. Therefore, the revealed LTLO phase is indispensable for understanding $\mathrm{La}_{2}-x \mathrm{Sr}_{x} \mathrm{CuO}_{4}$.
\end{abstract}

The observation of the superconductivity at $39 \mathrm{~K}$ in $\mathrm{MgB}_{2}$ [1] set a milestone for the superconducting transition temperature $\left(\mathrm{T}_{c}\right)$ in phonon-mediated conventional superconductors. 2] Recently, the highest $\mathrm{T}_{c}$ in the conventional superconductors was renewed again reaching $203 \mathrm{~K}$ in $\mathrm{H}_{3} \mathrm{~S}$ at high pressure. [3, 4] Very recently, the room-temperature superconductor, namely, superconducting at $288 \mathrm{~K}$, has been experimentally realized in carbonaceous sulfur hydride at $267 \mathrm{GPa},[5$, which remarks a great progress in the long search for the room-temperature superconductors under ambient conditions. Surprisingly, the underlying mechanism for the hydrogen-rich materials is still governed by the electronphonon coupling, [6, 7] proposed by Bardeen, Cooper, and Schrieffer (BCS) six decades ago. 2] On the other hand, the cuprate family, the representative of unconventional superconductors, is known for containing rich strong-correlation physics, where the theoretical prediction of $\mathrm{T}_{c}$ based on the electron-phonon pairing mechanism alone has not been successful in explaining the observed high $\mathrm{T}_{c} \cdot 8$. 10 Given that the Hg-based cuprates have been keeping the record of the highest $\mathrm{T}_{c}$ at ambient conditions, 11] the cuprate family is still on the list of the promising candidates for realizing room-temperature superconductivity without high pressure.

So far the key for realizing the room-temperature $\mathrm{T}_{c}$ in cuprates has not been found, but not all the properties of superconductivity in cuprates are too elusive to understand via the conventional mechanisms. [12] Given that the electron-phonon coupling is important for the carbonaceous sulfur hydride [5] and first-principles calculations have suggested the possession of strong electron-phonon coupling in cuprates, 13, 14 it is interesting to ask whether or not something related to the phonon properties has been missing in the studies of cuprates. In cuprates, high-temperature tetragonal (HTT), low-temperature tetragonal (LTT), lowtemperature orthorhombic (LTO), and low-temperature less-orthorhombic (LTLO) structures are ubiquitously observed, for example, in $\mathrm{La}_{2-x} \mathrm{Ba}_{x} \mathrm{CuO}_{4}$. 15 Generally speaking, the structural phase transitions can be explained by a soft-phonon model, [16 18 such as the first-order LTO $\rightarrow$ LTT and the second-order $\mathrm{HTT} \rightarrow \mathrm{LTO} \rightarrow \mathrm{LTLO} \rightarrow$ LTT transitions. But the actual transitions depend on the studied cuprates.

First-principles calculations without using advanced functionals cannot reproduce the observed insulating gaps in the undoped cuprates [19] but are capable of describing the phonon properties in the metallic phases. 13, 20.22 For the representative cuprate, $\mathrm{La}_{2-x} \mathrm{M}_{x} \mathrm{CuO}_{4}$, $\mathrm{La}_{2-x} \mathrm{Ba}_{x} \mathrm{CuO}_{4}$ exhibits rich phases and an anomalously deep depression at the superconducting phase boundary around $x=1 / 8$. [15, 23] In contrast, $\mathrm{La}_{2-x} \mathrm{Sr}_{x} \mathrm{CuO}_{4}$ exhibits a more standard dome-shaped boundary, and the HTT and LTO phases are the only major players in the phase diagram. 24 29] The HTT $\rightarrow$ LTO transition can be understood via the phonon softening in the HTT phase. [16, 21, 22, 30, $\mathrm{La}_{2-x} \mathrm{Sr}_{x} \mathrm{CuO}_{4}$ is then served as a relatively simple system for investigation. Nevertheless, the missing LTLO phase was observed at a seemingly exclusive doping level, namely, $x=0.12$. 31, 32. Recently, the presence of the LTLO structure, which is intimately related to the charge stripe order, has also been evidenced in $\mathrm{La}_{1.93} \mathrm{Sr}_{0.07} \mathrm{CuO}_{4}$ by neutron scatter- 

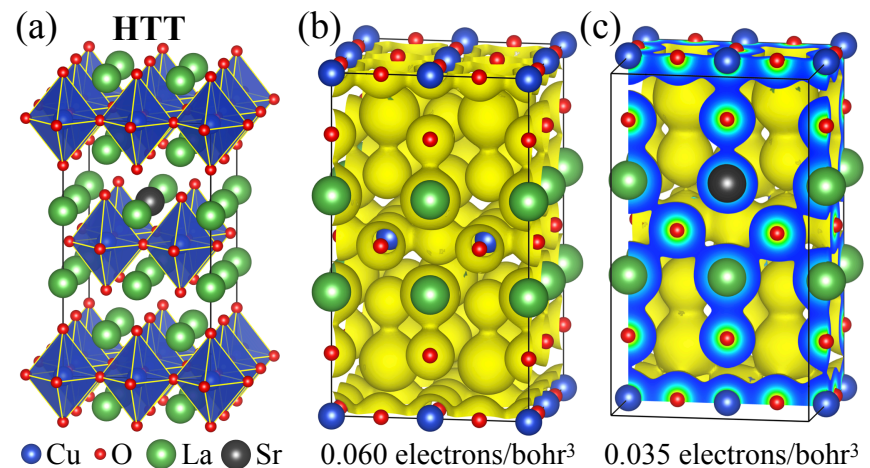

(d)

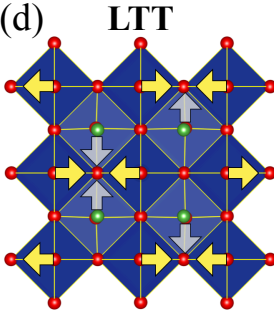

(e) LTO

(f) LTLO
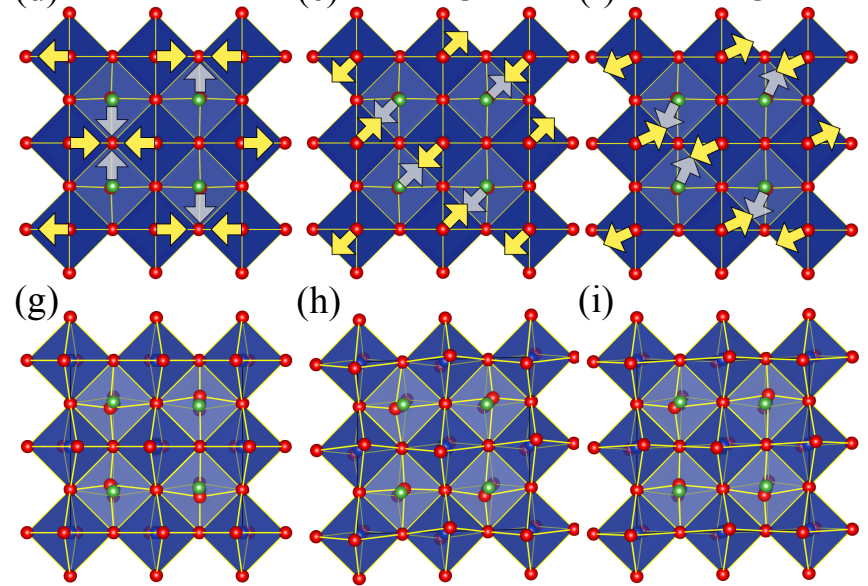

(h)

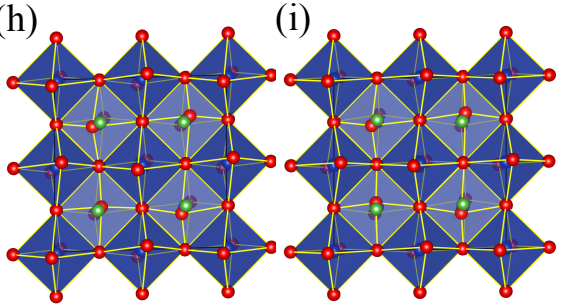

FIG. 1. (a) Side view of the high-temperature tetragonal (HTT) structure of $\mathrm{La}_{1.875} \mathrm{Sr}_{0.125} \mathrm{CuO}_{4}$, where $a=7.62 \AA$ and $c=13.22 \AA$. The $\mathrm{CuO}_{6}$ is presented by the octahedron. The isosurfaces of the charge density at 0.060 and 0.035 electrons/bohr ${ }^{3}$ are presented in (b) and (c), respectively. The tilting directions of the apical $\mathrm{O}$ atoms are indicated by the yellow (top layer) and grey (middle layer) arrows from the top view to describe the derived (d) low-temperature tetragonal (LTT), (e) low-temperature orthorhombic (LTO), and (f) low-temperature less orthorhombic (LTLO) structures from the HTT one. The calculated tilted octahedra of LTT, LTO, and LTLO structures are shown in (g), (h), and (i), respectively. The plots were generated using VESTA. 36]

ing experiments. 33 In this study, we will address the phonon instability in the nearly optimally doped LTO $\mathrm{La}_{1.875} \mathrm{Sr}_{0.125} \mathrm{CuO}_{4}$ based on the first-principles supercell calculations, which are different from previously adopted rigid-band and virtual-crystal approximations, 13, 22 and demonstrate that the LTLO phase is the ground state by quenching the magnetism, large-scale charge ordering, 29, 34, 35] and superconductivity.

The first-principles calculations based on the density functional theory (DFT) were performed using the OpenMX code, 37 where the generalized gradient approximation (GGA), norm-conserving pseudopotentials, and optimized pseudo-atomic basis functions were adopted.38 40] Three, three, three, and two optimized radial functions were allocated for the $s, p, d$, and $f$ orbitals, respectively, for each La atom with a cutoff radius of 8 bohr, denoted as La8.0-s3p3d3f2. For the Sr, $\mathrm{Cu}$, and $\mathrm{O}$ atoms, Sr10.0-s3p2d2f2, Cu6.0-s2p2d2, and O7.0-s2p2d1 were adopted, respectively. A cutoff energy of 500 Ry was used for numerical integrations and for the solution of the Poisson equation. $\mathrm{La}_{1.875} \mathrm{Sr}_{0.125} \mathrm{CuO}_{4}$ was studied via the unit cell containing a $\mathrm{Sr}$ atom, $8 \mathrm{Cu}$ atoms, $15 \mathrm{La}$ atoms, and $32 \mathrm{O}$ atoms, where one of the symmetrically equivalent La atoms was replaced by the Sr atom from the HTT, LTT, LTO, and LTLO structures with $I 4 / \mathrm{mmm}, P 4_{2} / \mathrm{ncm}$, Bmab, and Pccn symmetry, respectively. After the substitution, the structures were fully relaxed $(P 1)$ within DFT-GGA using a $4 \times 4 \times 2$ $k$-point sampling and all the forces are less than $10^{-4}$ Ha/bohr. The relaxed tilted octahedra are presented in Fig. 1. 41 The force constants needed for constructing the dynamical matrix were obtained from the $(2 \times 2 \times 1)$ supercell calculations (224 atoms).

The untilted $\mathrm{CuO}_{6}$ octahedra in the HTT structure, shown in Fig. 1 (a), serve as the building blocks to construct the LTT, LTO, and LTLO structures with different tilts, which are indicated by the arrows in Figs. 11(d), (e), and (f), respectively. The calculated structures within DFT-GGA are shown in Figs. 1 (g), (h), and (i), respectively, where the expected tilts can be recognized even with the presence of the $\mathrm{Sr}$ atom. The LTLO structure is apparently a mixture of the LTO and LTT ones, which supports a continuous LTLO-LTO or LTLO-LTT phase transition, whereas the direct LTO-LTT phase transition is first-order.10 Although the HTT and LTO phases are the only major players in $\mathrm{La}_{1-x} \mathrm{Sr}_{x} \mathrm{CuO}_{4}$, the total energies of the LTT, LTO, and LTLO phases relative to the HTT phase within DFT-GGA presented in Fig. 2 (a) reveal that the LTLO phase has the lowest energy. At $x=0$, DFT-GGA without considering magnetism cannot well describe $\mathrm{LaCuO}_{4}$, but for $x=0.125$, the long-range antiferromagnetic order has been destroyed so that DFT-GGA provides a good description for the normal-state $\mathrm{La}_{1.875} \mathrm{Sr}_{0.125} \mathrm{CuO}_{4}$. In Fig. 2 (b), the density of states is presented and exhibits a split feature in the LTLO and LTT $\mathrm{La}_{2} \mathrm{CuO}_{4}$. The split van Hove singularities near the Fermi level can offer an explanation for the presence of the pseudogaps based on the dynamic Jahn-Teller effect. 42 The splitting can be further introduced and enhanced in $\mathrm{La}_{1.875} \mathrm{Sr}_{0.125} \mathrm{CuO}_{4}$ due to the affected $\mathrm{CuO}_{6}$ tilting and Brillouin-zone periodicity. However, the intensity of the two peaks forming the gap at $-40 \mathrm{meV}$ is more prominent in the LTLO phase than that in the HTT phase. The Fermi arc 43] can be realized by unfolding the spectral weight 44 to a larger Brillouin zone at this energy, as illustrated in Fig. 2 (c).

To verify the dynamical stability of the HTT, LTO, and LTLO phases, the phonon properties are investigated and discussed here. The phonon dispersion in the HTT $\mathrm{La}_{1.875} \mathrm{Sr}_{0.125} \mathrm{CuO}_{4}$ is presented in Fig. 3 (a). One of the two degenerate vibrational modes at the lowest 

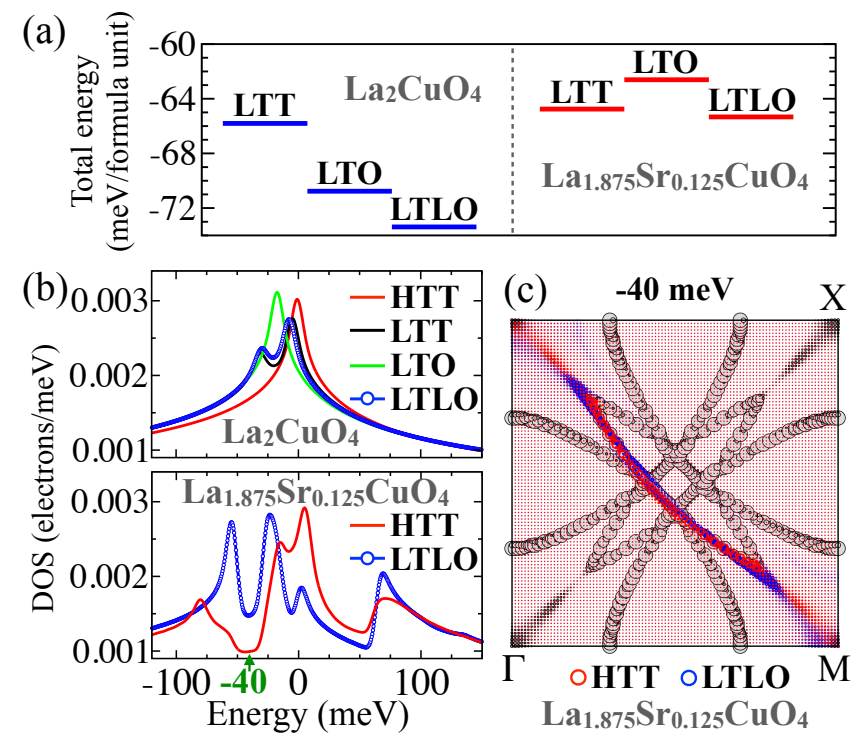

FIG. 2. (a) DFT-GGA energy levels of LTT, LTO, and LTLO phases relative to the total energy of HTT phase for $\mathrm{La}_{2} \mathrm{CuO}_{4}$ and $\mathrm{La}_{1.875} \mathrm{Sr}_{0.125} \mathrm{CuO}_{4}$, respectively. (b) Density of states (DOS) per formula unit for $k_{z}=0$ near the Fermi level (0 meV) in $\mathrm{La}_{2} \mathrm{CuO}_{4}$ and $\mathrm{La}_{1.875} \mathrm{Sr}_{0.125} \mathrm{CuO}_{4}$. (c) Spectral weight of Kohn-Sham orbitals in HTT (red circles) and LTLO (blue circles) $\mathrm{La}_{1.875} \mathrm{Sr}_{0.125} \mathrm{CuO}_{4}$ unfolded to the Brillouin zone of the primitive cell $(7$ atoms) with $\mathrm{M}:(0.5,0,0)$ and $\mathrm{X}:(0.5,0.5,0)$ in units of the reciprocal lattice vectors of the conventional cell (14 atoms). The original (folded) weight in HTT phase is presented by black circles.

(imaginary) frequency at $\mathrm{X}$ is in accordance with the guiding arrows for the HTT $\rightarrow$ LTO transition, shown in Fig. 1] (e), and the other mode has a 90-degree rotation. Presumably, the instability can be removed by following either one of the mutually orthogonal sets of eigendisplacements. To further verify the stability of the LTO phase, the phonon dispersion is presented in Fig. 3 (b). It can be found that imaginary-frequency modes still exist. Interestingly, the lowest-frequency vibrational mode at $\mathrm{X}$ is in accordance with the previously mentioned 90 degree-rotation mode. This indicates that the system can be further stabilized via additional tilting guided by the persisting phonon instability, which brings the LTO structure to the LTLO one. Finally, the phonon dispersion in the LTLO phase is shown in Fig. 3 (c), where no imaginary frequencies are present. The soft-phonon picture advocates the HTT $\rightarrow$ LTO $\rightarrow$ LTLO transitions in the normal-state $\mathrm{La}_{1.875} \mathrm{Sr}_{0.125} \mathrm{CuO}_{4}$ and is consistent with the calculated total energies.

The observed pseudogap in $\mathrm{La}_{2-x} \mathrm{Sr}_{x} \mathrm{CuO}_{4}$ can be related to the LTLO phase by considering the atoms displacing in a double well connecting the LTO and LTLO structures. As already illustrated in Fig. 2 (b), the split density of states in the LTLO structure could be responsible for the pseudogap. At lower temperatures, the structure intends to deviate from the cen-
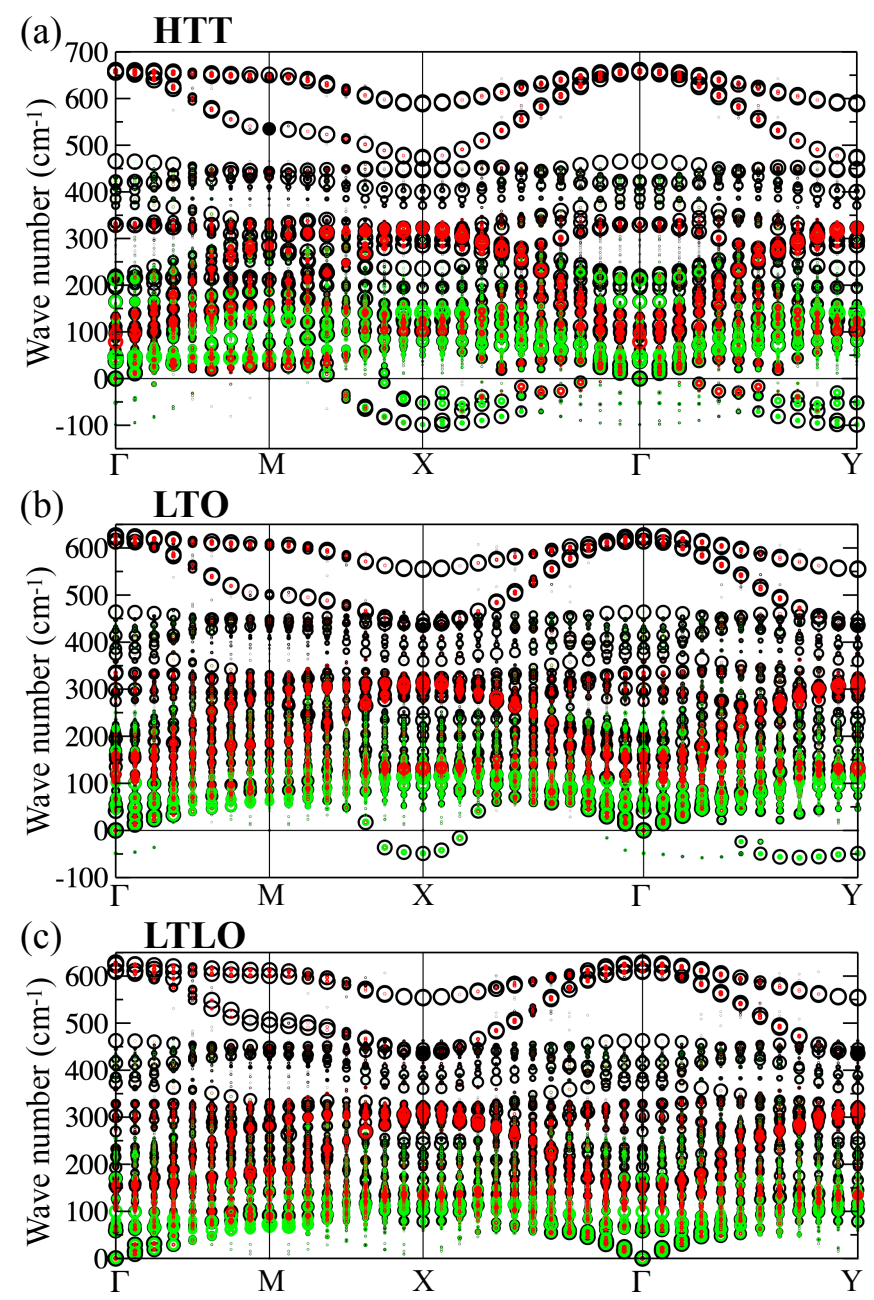

FIG. 3. DFT-GGA phonon dispersions in (a) HTT, (b) LTO, and (c) LTLO La ${ }_{1.875} \mathrm{Sr}_{0.125} \mathrm{CuO}_{4}$. The black circle represents the unfolded weight for each vibrational mode in the Brillouin zone of the conventional cell (14 atoms) with M:(0.5,0,0), $\mathrm{X}:(0.5,0.5,0)$, and $\mathrm{Y}:(-0.5,0.5,0)$. The radii of red (green) circles are proportional to the $\mathrm{Cu}(\mathrm{La})$ contribution.

ter of the double well and could develop charge-densitywave fluctuations. 29, 35 Since the LTLO phase may experience a continuous transition to the LTO or LTT phase, the observed stripe showing a mixture of LTOlike and LTT-like distortions 34 could have an intimate relationship with the LTLO structure. Our result also supports the mentioned role of the LTLO structure for the observed spin and charge fluctuations at high temperatures. 33. The LTT $\mathrm{La}_{1.875} \mathrm{Sr}_{0.125} \mathrm{CuO}_{4}$, which is also dynamically stable, has only slightly higher total energy than the LTLO one. The almost degenerate energy could also play a role for the depression around $x=0.125$ at the superconducting phase boundary. 29] Regarding that the energy difference between the LTO and LTLO phases is comparable with the superconducting gap, it is reasonable to argue that the absence of the LTLO phase 
at lower temperatures is due to the electron pairing that stabilizes the superconducting LTO phase in competing with the phonon-preferred LTLO phase.

We now reveal the major player for the phonon instability in the HTT and LTO phases. As presented by the radii of circles in Fig. 3 (a) for the HTT phase, both the $\mathrm{Cu}$ and La atoms are found to contribute to the imaginary-frequency branches. More specifically, the $\mathrm{Cu}$ atoms mainly contribute to the shallower part, whereas the La atoms are responsible for almost all the imaginaryfrequency modes. For the most unstable degenerate optical modes, whose frequency is $-98.5 \mathrm{~cm}^{-1}$ at $\mathrm{X}$, the atomic contribution is mainly from the $\mathrm{O}$ atoms. In the LTO phase, the $\mathrm{Cu}$ contribution disappears significantly in the imaginary-frequency branches, as shown in Fig. 3 (b), suggesting that the ingredients of phonon instability are composed of the La and $\mathrm{O}$ atoms. This finding implies that the structural phase transitions are driven by the La-related bonding. In fact, the isosurfaces of charge density in HTT $\mathrm{La}_{1.875} \mathrm{Sr}_{0.125} \mathrm{CuO}_{4}$, shown in Figs. 1](b) and (c) at 0.060 and 0.035 electrons $/$ bohr $^{3}$, respectively, clearly show that the apical $\mathrm{O}$ atoms only weakly bond to the center $\mathrm{Cu}$ atoms but strongly bond to the La atoms. Therefore, the intuitively understandable tilting of $\mathrm{CuO}_{6}$ octahedra, as shown in Fig. 1, does not describe the underlying mechanism for the structural phase transitions.

To demonstrate the roles of partial compositions in HTT $\mathrm{La}_{1.875} \mathrm{Sr}_{0.125} \mathrm{CuO}_{4}$ for the phonon instability, such as the structural stability of the $\mathrm{CuO}_{2}$ plane shown in Fig. 4 (a), we can rediagonalize the dynamical matrix by treating the irrelevant atoms in the unit cell as a rigid body without internal degrees of freedom of vibration, which is in accordance with the mobile block Hessian approximation. 45, 46] The force constants between the rigid clusters in the different unit cells are set to zero. The resultant dispersion of the $\mathrm{CuO}_{2}$ plane is shown in Fig. 4 (c), where no imaginary frequencies can be found. Alternatively, we can consider all the $\mathrm{Cu}$ and $\mathrm{Sr}$ atoms in the unit cell forming a heavy rigid cluster. As presented in Fig. 4 (c), the lowest-frequency branches in HTT $\mathrm{La}_{1.875} \mathrm{Sr}_{0.125} \mathrm{CuO}_{4}$ are well reproduced. This provides a solid support that the extracted structure composed of the La and $\mathrm{O}$ atoms, as shown in Fig. 4 (b), can well describe the phonon instability in the HTT phase.

In conclusion, the phonon properties in the nearly optimally doped $\mathrm{La}_{1.875} \mathrm{Sr}_{0.125} \mathrm{CuO}_{4}$ have been studied within DFT-GGA, where the La-O composition is identified to be responsible for the phonon instability giving rise to the $\mathrm{HTT} \rightarrow \mathrm{LTO} \rightarrow \mathrm{LTLO}$ transitions, while the superconducting $\mathrm{CuO}_{2}$ layer itself is dynamically stable. One of the softest doubly degenerate modes in the HTT phase triggers the HTT $\rightarrow$ LTO transition, and the other mode still persists in the LTO phase to further drive the LTO $\rightarrow$ LTLO transition. The calculated total energies also support that the LTLO phase is the ground state at $x=0$ and $1 / 8$ by quenching the magnetism, charge- (a)

(b)
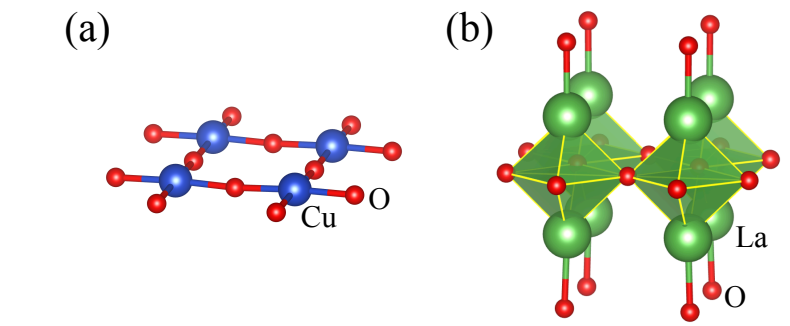

(c)

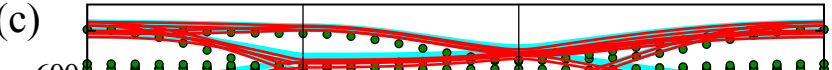

$600+8$ \& \& 1 i

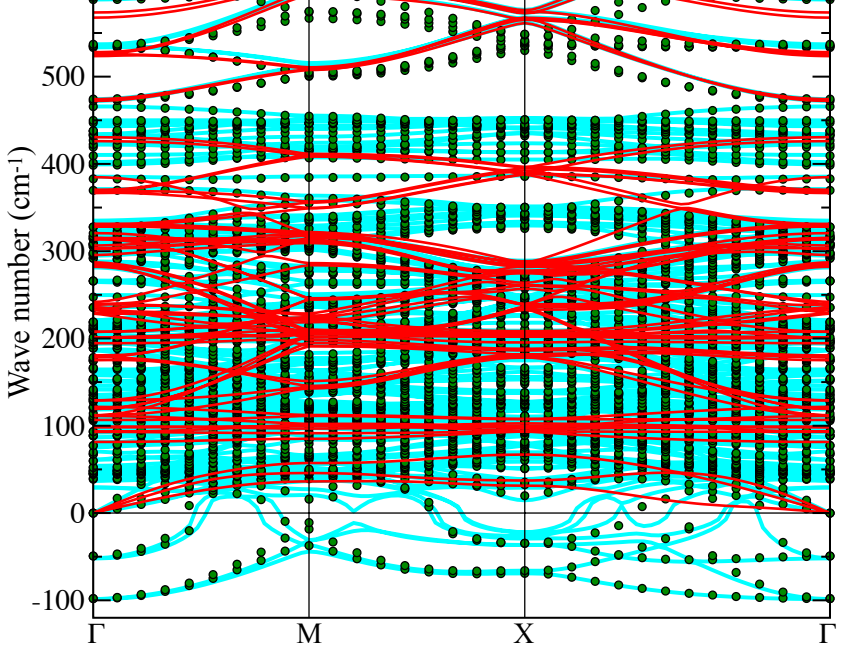

FIG. 4. (a) The $\mathrm{CuO}_{2}$ layer and (b) the extended octahedra composed of $\mathrm{La}$ and $\mathrm{O}$ atoms in $\mathrm{HTT} \mathrm{La}_{1.875} \mathrm{Sr}_{0.125} \mathrm{CuO}_{4}$. (c) Reconstructed phonon dispersions of the (a) planar $\mathrm{CuO}_{2}$ and (b) extracted octahedra are plotted by red curves and green circles, respectively. The original supercell phonon dispersion with M: $(0.5,0,0)$ and $\mathrm{X}:(0.5,0.5,0)$ is presented by cyan curves.

density-wave order, and superconductivity. We propose that the LTO structure can be described by a displacive model involving the LTLO $\leftrightarrow$ LTO $\leftrightarrow$ LTLO displacements. The exhibited $\sim 20 \mathrm{meV}$ splitting in the density of states in the LTLO phase can be related to the observed pseudogap. At low temperatures, we expect that the LTLO phase encounters a fierce competition with the observed LTO phase, which can gain more energy from the electron pairing in the superconducting state and therefore stabilize the LTO structure. This indicates that the calculation of $\mathrm{T}_{c}$ via the electron-phonon coupling cannot be decoupled from the superconducting state. Finally, we emphasize that the missing LTLO phase provides an indispensable ingredient in describing $\mathrm{La}_{2-x} \mathrm{Sr}_{x} \mathrm{CuO}_{4}$.

The calculations were carried out using the facilities in JAIST and Tamkang University. Chi-Cheng Lee acknowledges the Ministry of Science and Technology of Taiwan for financial support under contract No. MOST 108-2112-M-032-010-MY2. 
[1] J. Nagamatsu, N. Nakagawa, T. Muranaka, Y. Zenitani, and J. Akimitsu, Nature 410, 63 (2001).

[2] J. Bardeen, L. N. Cooper, and J. R. Schrieffer, Phys. Rev. 108, 1175 (1957)

[3] A. P. Drozdov, M. I. Eremets, I. A. Troyan, V. Ksenofontov, and S. I. Shylin, Nature 525, 73 (2015).

[4] D. Duan, Y. Liu, F. Tian, D. Li, X. Huang, Z. Zhao, H. Yu, B. Liu, W. Tian, and T. Cui, Sci. Rep. 4, 6968 (2014)

[5] E. Snider, N. Dasenbrock-Gammon, R. McBride, M. Debessai, H. Vindana, K. Vencatasamy, K. V. Lawler, A. Salamat, and R. P. Dias, Nature 586, 373 (2020)

[6] D. Y. Kim, R. H. Scheicher, H.-k. Mao, T. W. Kang, and R. Ahuja, PNAS 107, 2793 (2010).

[7] K. Tanaka, J. S. Tse, and H. Liu, Phys. Rev. B 96, 100502 (2017)

[8] A. Damascelli, Z. Hussain, and Z.-X. Shen, Rev. Mod. Phys. 75, 473 (2003)

[9] P. A. Lee, N. Nagaosa, and X.-G. Wen, Rev. Mod. Phys. 78, 17 (2006).

[10] N. Plakida, High-Temperature Cuprate Superconductors: Experiment, Theory, and Applications, Vol. 166 (Springer Science \& Business Media, 2010).

[11] A. Schilling, M. Cantoni, J. D. Guo, and H. R. Ott, Nature 363, 56 (1993).

[12] C. Berthod, I. Maggio-Aprile, J. Bruér, A. Erb, and C. Renner, Phys. Rev. Lett. 119, 237001 (2017)

[13] H. Krakauer, W. E. Pickett, and R. E. Cohen, Phys. Rev. B 47, 1002 (1993)

[14] Z. P. Yin, A. Kutepov, and G. Kotliar, Phys. Rev. X 3, 021011 (2013)

[15] M. Hücker, M. v. Zimmermann, G. D. Gu, Z. J. Xu, J. S. Wen, G. Xu, H. J. Kang, A. Zheludev, and J. M. Tranquada, Phys. Rev. B 83, 104506 (2011).

[16] J. D. Axe, A. H. Moudden, D. Hohlwein, D. E. Cox, K. M. Mohanty, A. R. Moodenbaugh, and Y. Xu, Phys. Rev. Lett. 62, 2751 (1989).

[17] A. Y. Cherny, Physica C 244, 129 (1995).

[18] H. Kimura, Y. Noda, H. Goka, M. Fujita, K. Yamada, and G. Shirane, Journal of the Physical Society of Japan 74, 445 (2005)

[19] J. W. Furness, Y. Zhang, C. Lane, I. G. Buda, B. Barbiellini, R. S. Markiewicz, A. Bansil, and J. Sun, Commun. Phys. 1, 1 (2018)

[20] R. E. Cohen, W. E. Pickett, and H. Krakauer, Phys. Rev. Lett. 62, 831 (1989)

[21] C.-Z. Wang, R. Yu, and H. Krakauer, Phys. Rev. B 59, 9278 (1999)

[22] A. Tavana, M. Akhavan, and C. Draxl, Physica C 517, 20 (2015)

[23] M. Fujita, H. Goka, K. Yamada, and M. Matsuda, Phys.
Rev. Lett. 88, 167008 (2002).

[24] P. Day, M. Rosseinsky, K. Prassides, W. I. F. David, O. Moze, and A. Soper, J. Phys. C: Solid State Phys. 20, L429 (1987).

[25] H. Takagi, T. Ido, S. Ishibashi, M. Uota, S. Uchida, and Y. Tokura, Phys. Rev. B 40, 2254 (1989).

[26] B. Keimer, N. Belk, R. J. Birgeneau, A. Cassanho, C. Y. Chen, M. Greven, M. A. Kastner, A. Aharony, Y. Endoh, R. W. Erwin, and G. Shirane, Phys. Rev. B 46, 14034 (1992)

[27] H. Takahashi, H. Shaked, B. A. Hunter, P. G. Radaelli, R. L. Hitterman, D. G. Hinks, and J. D. Jorgensen, Phys. Rev. B 50, 3221 (1994)

[28] J. L. Sarrao, D. Mandrus, A. Migliori, Z. Fisk, I. Tanaka, H. Kojima, P. C. Canfield, and P. D. Kodali, Phys. Rev. B 50, 13125 (1994).

[29] T. P. Croft, C. Lester, M. S. Senn, A. Bombardi, and S. M. Hayden, Phys. Rev. B 89, 224513 (2014).

[30] P. Böni, J. D. Axe, G. Shirane, R. J. Birgeneau, D. R. Gabbe, H. P. Jenssen, M. A. Kastner, C. J. Peters, P. J. Picone, and T. R. Thurston, Phys. Rev. B 38, 185 (1988).

[31] Y. Koyama, Y. Wakabayashi, K. Ito, and Y. Inoue, Phys. Rev. B 51, 9045 (1995)

[32] Y. Horibe, Y. Inoue, and Y. Koyama, Phys. Rev. B 61, $11922(2000)$

[33] H. Jacobsen, I. A. Zaliznyak, A. T. Savici, B. L. Winn, S. Chang, M. Hücker, G. D. Gu, and J. M. Tranquada, Phys. Rev. B 92, 174525 (2015).

[34] A. Bianconi, N. L. Saini, A. Lanzara, M. Missori, T. Rossetti, H. Oyanagi, H. Yamaguchi, K. Oka, and T. Ito, Phys. Rev. Lett. 76, 3412 (1996).

[35] Y. Wang, Y. Zhong, Z. Luo, M. Liao, R. Wang, Z. Dou, Q. Zhang, D. Zhang, L. Gu, C.-L. Song, et al., npj Quantum Mater. 4, 1 (2019)

[36] K. Momma and F. Izumi, J. Appl. Crystallogr. 44, 1272 (2011)

[37] The code, OpenMX, pseudo-atomic basis functions, and pseudopotentials are available on a website (http://www.openmx-square.org/).

[38] J. P. Perdew, K. Burke, and M. Ernzerhof, Phys. Rev. Lett. 77, 3865 (1996)

[39] I. Morrison, D. M. Bylander, and L. Kleinman, Phys. Rev. B 47, 6728 (1993).

[40] T. Ozaki, Phys. Rev. B 67, 155108 (2003)

[41] The relaxed structures of $\mathrm{La}_{1.875} \mathrm{Sr}_{0.125} \mathrm{CuO}_{4}$ can be found in the supplementary materials.

[42] R. S. Markiewicz, J. Phys. Chem. Solids 58, 1179 (1997).

[43] T. Yoshida et al., Phys. Rev. B 74, 224510 (2006).

[44] C.-C. Lee, Y. Yamada-Takamura, and T. Ozaki, Journal of Physics: Condensed Matter 25, 345501 (2013)

[45] A. Ghysels, D. V. Neck, V. V. Speybroeck, T. Verstraelen, and M. Waroquier, J. Chem. Phys. 126, 224102 (2007)

[46] R. Terrett, R. Stranger, T. Frankcombe, and R. J. Pace, Phys. Chem. Chem. Phys. 19, 6654 (2017) 